\title{
Methanol masers reveal the magnetic field of the high-mass protostar IRAS 18089-1732
}

\author{
D. Dall'Olio ${ }^{1}$, W. H. T. Vlemmings ${ }^{1}$, G. Surcis ${ }^{2}$, H. Beuther ${ }^{3}$, B. Lankhaar ${ }^{1}$, M. V. Persson ${ }^{1}$, \\ A. M. S. Richards ${ }^{4}$, and E. Varenius ${ }^{1}$

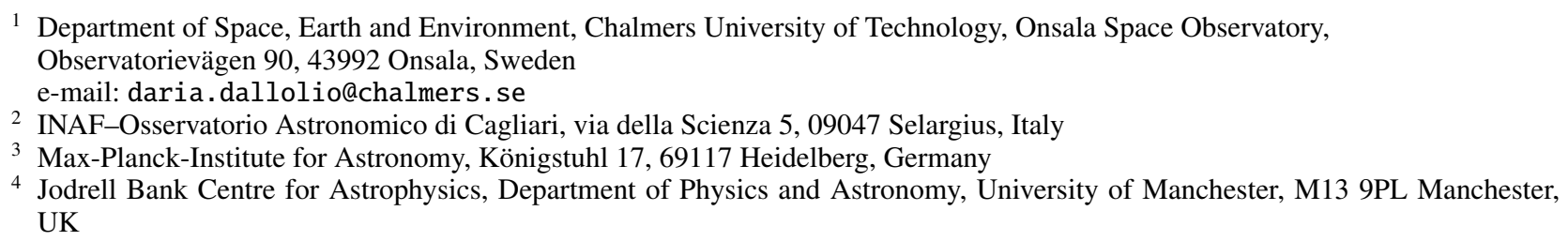

Received 1 June 2017 / Accepted 8 August 2017

\begin{abstract}
Context. The importance of the magnetic field in high-mass-star formation is not yet fully clear and there are still many open questions concerning its role in the accretion processes and generation of jets and outflows. In the past few years, masers have been successfully used to probe the magnetic field morphology and strength at scales of a few au around massive protostars, by measuring linear polarisation angles and Zeeman splitting. The massive protostar IRAS 18089-1732 is a well studied high-mass-star forming region, showing a hot core chemistry and a disc-outflow system. Previous SMA observations of polarised dust revealed an ordered magnetic field oriented around the disc of IRAS 18089-1732.

Aims. We want to determine the magnetic field in the dense region probed by $6.7 \mathrm{GHz}$ methanol maser observations and compare it with observations in dust continuum polarisation, to investigate how the magnetic field in the compact maser region relates to the large-scale field around massive protostars.

Methods. We reduced MERLIN observations at $6.7 \mathrm{GHz}$ of IRAS 18089-1732 and we analysed the polarised emission by methanol masers.

Results. Our MERLIN observations show that the magnetic field in the $6.7 \mathrm{GHz}$ methanol maser region is consistent with the magnetic field constrained by the SMA dust polarisation observations. A tentative detection of circularly polarised line emission is also presented.

Conclusions. We found that the magnetic field in the maser region has the same orientation as in the disk. Thus the large-scale field component, even at the au scale of the masers, dominates over any small-scale field fluctuations. We obtained, from the circular polarisation tentative detection, a field strength along the line of sight of $5.5 \mathrm{mG}$ which appeared to be consistent with the previous estimates.
\end{abstract}

Key words. magnetic fields - masers - stars: formation - stars: massive - polarization

\section{Introduction}

The role of magnetic fields during the formation of highmass stars is not yet fully understood. As in the case of lowmass star formation, simulations have shown that the magnetic field appears to prevent fragmentation around massive protostars (Peters et al. 2011; Myers et al. 2013) to influence accretion and to drive feedback phenomena such as collimated outflows and jets (Seifried et al. 2011, 2012). Both the core accretion model (e.g. McKee \& Tan 2003; Banerjee \& Pudritz 2007) and the competitive accretion model (e.g. Bonnell \& Bate 2006) need observational constraints on magnetic fields to properly investigate their effect on the high-mass star formation process (Tan et al. 2014).

Several fine-tuned models have shown that some detailed and specific magneto-hydrodynamic (MHD) configurations, yet to be tested observationally, can explain observed morphologies (e.g. Krumholz et al. 2013; Li et al. 2014; Seifried et al. 2015, and references therein). A typical example is a rotating Keplerian disc around a protostar. While several circumstellar discs have been observed around massive protostars (Cesaroni et al. 2006, 2007; Beltrán \& de Wit 2016), theoretical studies have found it difficult to form such discs due to strong magnetic braking which removes most of the angular momentum from the circumstellar gas. This is the so-called magnetic braking catastrophe in disc formation (Mestel \& Spitzer 1956; Allen et al. 2003; Mellon \& Li 2008; Li et al. 2011, 2013). Only by inserting non-ideal MHD effects such as Ohmic dissipation or ambipolar diffusion, or the combined action of both, is it possible to overcome the apparent conflict between observations and simulations (Machida et al. 2014; Zhao et al. 2016). Moreover, Zhao et al. (2016) have shown that chemistry and microscopic physical processes, including advection of gas phase and grain species as well as grain evolution, must also be inserted in non-ideal MHD simulations to obtain a more realistic picture of the behaviour of a strongly magnetised core. However, it is not yet clear which process dominates between Ohmic dissipation and ambipolar diffusion; it probably depends on the magnitude of the initial magnetic energy density relative to the gravitational and turbulent energy density and the initial magnetic field configuration. 
Thus, we need to probe the magnetic field at small scales. The identification of high-mass protostars is however extremely complicated due to their fast evolution, and their location inside distant, dense, and dark clusters.

The first full polarisation observations of $6.7 \mathrm{GHz}$ methanol masers were made by Ellingsen (2002). Thereafter, Green et al. (2007), Vlemmings et al. (2006, 2010), Vlemmings (2008), Dodson \& Moriarty (2012) and Surcis et al. (2012, 2014b, 2015) have demonstrated that maser emission (from e.g. methanol and water) allows us to probe magnetic fields. Through the study of linear and circular polarised emission, it is possible to obtain the strength, morphology, and evolution of the magnetic field on scale comparable to circumstellar discs $(\sim 100 \mathrm{au})$. However, this has been done only in a limited number of cases which still prevents building a complete picture of the role played by magnetic fields. Most importantly, what is still lacking is more observational evidence that the magnetic field at small scales probed by masers represents the field at larger scales, probed, for example, by the dust, and not small-scale fluctuations. Currently, few observations of both masers and dust polarisation exist towards the same regions (e.g. Surcis et al. 2014b).

The high-mass protostar IRAS 18089-1732 is particularly important because observations of dust emission have already shown the structure of the magnetic field at large scales ( $\sim 5000 \mathrm{au}$, Beuther et al. 2010). In this paper, we investigate its small-scale magnetic field, by analysing a three-epoch MultiElement Radio Linked Interferometer Network (MERLIN) observation of the $6.7 \mathrm{GHz} \mathrm{CH} \mathrm{CH}_{3} \mathrm{OH}$ (methanol) maser generated in the same region of the disc. We use large scale to refer to arcsec scales within a $\sim 4^{\prime \prime}$ region centred on IRAS 18089-1732, and small scale for scales for a typical maser region size of $\sim 10$ mas within the same region. We present the first polarised map of the masers for IRAS 18089-1732 and we show that the small-scale magnetic field probed by the masers is consistent with the largescale magnetic field traced by the dust.

The structure of this paper is as follows. We introduce IRAS 18089-1732 in Sect. 2. We describe the observations and data analysis in Sect. 3. We present our result in Sect. 4 and discuss them in Sect. 5. In Sect. 6 we give our conclusions and future perspectives.

\section{The case of IRAS 18089-1732}

IRAS 18089-1732 (hereafter IRAS 18089) is a well studied protostar presenting a velocity $v_{\mathrm{lsr}}=33.8 \mathrm{~km} \mathrm{~s}^{-1}$ (Beuther et al. 2005) and located at a distance of $2.34 \mathrm{kpc}$ (Xu et al. 2011). It has a luminosity $L=1.3 \times 10^{4} L_{\odot}$ (Sridharan et al. 2002; rescaled to the adopted distance) and a gaseous mass $M \sim 1000 M_{\odot}$ estimated from single-dish millimetre continuum observations (Beuther et al. 2002; also rescaled to the adopted distance).

IRAS 18089 presents the typical chemistry of "hot cores" with a line forest profile and strong molecular emission, coming from, for example, $\mathrm{HCOOCH}_{3}, \mathrm{H}_{2} \mathrm{~S}, \mathrm{SO}$, and $\mathrm{SO}_{2}$ (Beuther et al. 2004a; Isokoski et al. 2013). The source is also a well-known disc-outflow system. Submillimiter Array (SMA) observed a $\mathrm{SiO}(5-4)$ molecular outflow in an approximately north-south direction (Beuther et al. 2004b) and showed rotational signatures in many molecular lines typical of an accreting disc in the dense gas perpendicular to the outflow (Beuther et al. 2005; Zapata et al. 2006; Beuther \& Walsh 2008). Moreover, the Goldreich-Kylafis effect (Goldreich \& Kylafis 1981, 1982) was detected for the $\mathrm{CO}(3-2)$ transition by Beuther et al. (2010), revealing a linear polarisation fraction up to $8 \%$. Furthermore, Beuther et al. (2010) showed that the magnetic field structure is largely aligned with the jet-outflow orientation, from the smaller scales of the core to the larger scales of the outflow. In addition, Beuther et al. (2010) estimated a magnetic field strength in the plane of the sky of $B_{\text {pos }} \sim 11 \mathrm{mG}$ at a core density of $5 \times 10^{7} \mathrm{~cm}^{-3}$. This value was estimated from the analysis of the polarised dust continuum emission observed with the SMA at $880 \mu \mathrm{m}$.

Vlemmings (2008) derived a comparable line-of-sight magnetic field strength $B_{\text {los }} \sim 8 \mathrm{mG}$ from the Zeeman splitting of the $6.7 \mathrm{GHz} \mathrm{CH}_{3} \mathrm{OH}$ maser line, at densities $>10^{6} \mathrm{~cm}^{-3}$. Therefore Beuther et al. (2010) finally estimated a total magnetic field strength $B_{\mathrm{tot}} \sim \sqrt{B_{\mathrm{pos}}^{2}+B_{\mathrm{los}}^{2}} \sim 14 \mathrm{mG}$, which is of the same order of measurements made by Girart et al. (2009), Surcis et al. (2009), and Vlemmings et al. (2010) for similar sources. However the estimation by Beuther et al. (2010) was made using an extrapolation of the $g$-factor, obtained from measurements of $25 \mathrm{GHz}$ methanol transitions. This may result in a derived magnetic field strength that is larger by an order of magnitude with respect to the true field strength, as described by Vlemmings et al. (2011).

Walsh et al. (1998) provided a map of IRAS 18089 masers from Australia Telescope Compact Array (ATCA) observations and obtained relative and absolute positions with an accuracy of around $0.05^{\prime \prime}$ and $1^{\prime \prime}$ respectively. Goedhart et al. (2009), monitoring the variability in IRAS 18089 , reported a periodicity of the flares maxima of around $29.5 \pm 0.1$ days, derived after 9 years of observations with Hartebeesthoek Radio Astronomy Observatory $26 \mathrm{~m}$ telescope.

\section{Observations and data reduction}

IRAS 18089-1732 was observed by MERLIN at $6.7 \mathrm{GHz}$, in March, April, and July 2008, and the data were stored in three datasets. The observations were obtained with a single spectral window with 255 channels, covering a bandwidth of $249 \mathrm{kHz}$ in March and April, and a bandwidth of $498 \mathrm{kHz}$ in July. The total on-source observing time was $28 \mathrm{~h}, 7 \mathrm{~h}$ in March and July, and $14 \mathrm{~h}$ in April. Six antennas were used for the first two observations, and five antennas for the last observation when Defford was not included. The longest baseline of MERLIN is $217 \mathrm{~km}$. The observational details are reported in Table 1.

The datasets were reduced using the Astronomical Image Processing Software (AIPS version Dec. 2016), and the calibration was performed using 3C 286, MRC 1757-150 and 3C 84. $3 \mathrm{C} 286$ was chosen as flux and polarisation angle calibrator, MRC 1757-150 as the phase calibrator, and 3C 84 was used to calibrate the bandpass. Since 3C 286 is known to be resolved, for the flux calibration we used a model of 3C 286 provided by the MERLIN database ${ }^{1}$.

The March and July observations each consisted of a single run, while two runs were performed in April, over two consecutive days. In March and July the phase calibrator and the target were observed alternately for respectively 2 and $6 \mathrm{~min}$, while in April for respectively 1 and $8 \mathrm{~min}$, over a total time of about $7 \mathrm{~h}$ per run. In the second run the phase calibration failed due to an unusual observational strategy, so we used a model based on the strongest maser of the first run to self calibrate the second run, using a solution interval of $10 \mathrm{~min}$. For an overview of the calibration strategy we refer to the MERLIN User Guide ${ }^{2}$. In Table 2 we report the fluxes of each calibrator. The Local Standard of

\footnotetext{
http://www.e-merlin.ac.uk/data-red/

http://www.e-merlin.ac.uk/user-guide/
} 
Table 1. Observational details for IRAS 18089-1732.

\begin{tabular}{ccccccc}
\hline \hline $\begin{array}{c}\text { Observation } \\
\text { date }\end{array}$ & $\begin{array}{c}\text { Polarisation } \\
\text { mode }\end{array}$ & $\begin{array}{c}\text { Bandwidth }^{a} \\
(\mathrm{kHz})\end{array}$ & $\begin{array}{c}\text { Channel } \\
\text { spacing }\left(\mathrm{km} \mathrm{s}^{-1}\right)\end{array}$ & $\begin{array}{c}\text { Beam size } \\
(\operatorname{arcsec} \times \operatorname{arcsec})\end{array}$ & $\begin{array}{c}\text { Position angle } \\
\left({ }^{\circ}\right)\end{array}$ & $\begin{array}{c}\mathrm{rms}^{b} \\
\left(\mathrm{Jy} \mathrm{beam}^{-1}\right)\end{array}$ \\
\hline 13 March 2008 & RR, LL, RL, LR & 249 & $\sim 0.05$ & $0.18 \times 0.03$ & 10.11 & 0.03 \\
7-8 April 2008 & RR, LL, RL, LR & 249 & $\sim 0.05$ & $0.18 \times 0.03$ & 12.39 & 0.02 \\
4 July 2008 & RR, LL & 498 & $\sim 0.09$ & $0.24 \times 0.08$ & -16.34 & 0.04 \\
\hline
\end{tabular}

Notes. ${ }^{(a)}$ The spectral window included 255 channels. ${ }^{(b)}$ rms on the line-free channels.

Table 2. Fluxes in Jy of the calibrators used in the three epochs.

\begin{tabular}{cccc}
\hline \hline $\begin{array}{c}\text { Observation } \\
\text { date }\end{array}$ & $\begin{array}{c}\text { MRC 1757-150 } \\
\text { (observed) }\end{array}$ & $\begin{array}{c}\text { 3C 84 } \\
\text { (observed) }\end{array}$ & $\begin{array}{c}\text { 3C 286 } \\
\text { (model) }\end{array}$ \\
\hline 13 March 2008 & 0.14 & 10.32 & 5.70 \\
7-8 April 2008 & 0.14 & 14.26 & 5.70 \\
4 July 2008 & 0.15 & 15.14 & 5.70 \\
\hline
\end{tabular}

Rest (LSR) correction was applied to ensure constant velocity in the target frame.

Self-calibration was performed on the brightest maser feature of each epoch, using a solution interval of $30 \mathrm{~s}$. The peak flux density of the brightest maser features was $\sim 70 \mathrm{Jy}^{\text {beam }}{ }^{-1}$ in March, $\sim 122 \mathrm{Jy} \mathrm{beam}^{-1}$ in April and $\sim 75 \mathrm{Jy}^{\text {beam }}{ }^{-1}$ in July, always at a velocity $V_{\mathrm{lsr}}=39.2 \mathrm{~km} \mathrm{~s}^{-1}$. The spectra of the three epochs, obtained by summing all the pixels in the image for each channel, are plotted in Fig. 1, and brightest maser feature spectra for the three epochs are shown in Fig. 2.

We extracted the $I, Q, U$, and $V$ cubes using the task IMAGR (with an image size of $6.14^{\prime \prime} \times 6.14^{\prime \prime}$ and a cell size of $0.006^{\prime \prime}$ ). As reported in Table 1, IRAS 18089 was observed in full polarisation mode only during March and April, while the July observations were in dual circular polarisation only. Thus, the linear polarisation calibration was done only for these two epochs. The rms noise in the line-free channels is reported in Table 1; however the noise increases in the channels with strongest maser features where the noise is dominated by the dynamic range limitations.

In March, the noise increased up to $\sim 0.2 \mathrm{Jy}^{-1}$ beam $^{-1}$ in $I, \sim 0.17 \mathrm{Jy} \mathrm{beam}^{-1}$ in $Q, \sim 0.13 \mathrm{Jy} \mathrm{beam}^{-1}$ in $U$, and $\sim 0.05 \mathrm{Jy} \mathrm{beam}^{-1}$ in $V$. In April, the noise reached $\sim 0.2 \mathrm{Jy} \mathrm{beam}^{-1}$ in $I, \sim 0.25 \mathrm{Jy}$ beam $^{-1}$ in $Q, \sim 0.15 \mathrm{Jy} \mathrm{beam}^{-1}$ in $U$, and $\sim 0.07 \mathrm{Jy}$ beam ${ }^{-1}$ in $V$. In July we only have maps in Stokes $I$ and $V$, and in the channels with the strongest features, the noise increased up to $\sim 0.12 \mathrm{Jy} \mathrm{beam}^{-1}$ and $\sim 0.06 \mathrm{Jy} \mathrm{beam}^{-1}$, respectively.

In all epochs we estimated residual leakages of less than the rms noise.

We combined the $\mathrm{U}$ and $\mathrm{Q}$ datacubes to produce cubes of polarised intensity $\left(\mathrm{POLI}=\sqrt{Q^{2}+U^{2}}\right)$ and polarisation angle $(\operatorname{POLA}=1 / 2 \times \operatorname{atan}(U / Q))$. The error on POLA includes the formal error due to the thermal noise (Wardle \& Kronberg 1974). This error is given by $\sigma_{\mathrm{POLA}}=0.5\left(\sigma_{\mathrm{P}} / \mathrm{POLI}\right) \times\left(180^{\circ} / \pi\right)$, where $\sigma_{\mathrm{P}}$ is the rms of POLI. We compared the linear polarization angles for 3C 286 measured in our observations with the angles reported by the NRAO in the Polarization Calibration Database ${ }^{3}$. 3C 286 is a standard well-known calibrator with a stable polarisation angle of $33.0^{\circ}$ at $6.7 \mathrm{GHz}$. After the calibration, we found angles of $38^{\circ} \pm 5^{\circ}$ and $36^{\circ} \pm 3^{\circ}$ in March and April respectively, which are consistent with the angle given in the database.

\footnotetext{
3 www.vla.nrao.edu/astro/calib/polar/2008
}

\section{Results}

The maser identification procedure follows the method already described in Surcis et al. (2011). The maser finder code looks for maser features inside the datacube, velocity channel by velocity channel. The code recognises a maser spot when the signal to noise ratio $(\mathrm{S} / \mathrm{N})$ of the candidate (using the local $\mathrm{rms}$ ) is greater than a predefined value. In the case of IRAS 18089 , we adopted a lower limit of 8 . A Gaussian fit is performed for each maser spot using the AIPS task IMFIT; the code generates a table containing parameters such as positions, velocities, and peak flux densities for each spot. A maser feature is identified only when the maser spots coincide spatially in at least three consecutive velocity channels. In March and April we found nine masers, while in July we found seven masers.

In Tables 3-5 we present the $6.7 \mathrm{GHz}$ methanol maser features detected during the three epochs and that fulfil the criteria described above. Not all features show up in all the three epochs. Feature F.08 was observed only in March, while F.10 was observed only in April, and F.11 only in July. The features maintain the same names when they were observed across the three epochs.

We found a systematic shift of the positions of the brightest masers between the three epochs but in each case this is less than the beam size. At the distance of IRAS 18089, proper motions in a few months would be negligible. In the tables, all maser positions are given as offsets relative to the position of F.01 at that epoch. The absolute position of F.01 in March is $\alpha_{2000}=18^{\mathrm{h}} 11^{\mathrm{m}} 51.3954^{\mathrm{s}} \pm 0.0001^{\mathrm{s}}, \delta_{2000}=-17^{\circ} 31^{\prime} 29.92^{\prime \prime} \pm$ $0.01^{\prime \prime}$, in April it is $\alpha_{2000}=18^{\mathrm{h}} 11^{\mathrm{m}} 51.3929^{\mathrm{s}} \pm 0.0003^{\mathrm{s}}, \delta_{2000}=$ $-17^{\circ} 31^{\prime} 29.86^{\prime \prime} \pm 0.03^{\prime \prime}$ and in July it is $\alpha_{2000}=18^{\mathrm{h}} 11^{\mathrm{m}} 51.399^{\mathrm{s}} \pm$ $0.002^{\mathrm{s}}, \delta_{2000}=-17^{\circ} 31^{\prime} 29.93^{\prime \prime} \pm 0.1^{\prime \prime}$. At $-17^{\circ}$ declination the errors are dominated by the phase fluctuations. Other contributions to the position accuracy include uncertainty in the position of the telescope, noise-based errors in component fitting, and uncertainty in the phase reference position. The weighted average position of the brightest maser F.01 is $\alpha_{2000}=18^{\mathrm{h}} 11^{\mathrm{m}} 51.3949^{\mathrm{s}} \pm$ $0.0005^{\mathrm{s}}, \delta_{2000}=-17^{\circ} 31^{\prime} 29.92^{\prime \prime} \pm 0.01^{\prime \prime}$.

In March, the offset of F.01 from the average position was -33 mas in RA and 4.3 mas in Dec. In April the offset was -68.6 mas in RA and 64.0 mas in Dec. In July the offset was 16.0 mas in RA and -8.1 mas in Dec. The peak flux densities reported in the tables were obtained from a Gaussian fit to the image of the brightest emission channel of the maser feature. The error on the relative positions reported in the tables was computed following Reid et al. (1988).

No cross-hand polarisation products were correlated for the July observation, and hence no linear polarisation values were obtained. Therefore we could only perform the linear polarisation analysis for the March and April datasets. Four features in March and six features in April showed linear polarisation for which we measured the median linear polarisation fraction $\left(P_{1}\right)$ and the median linear polarisation angle $(\chi)$ across the spectrum. 


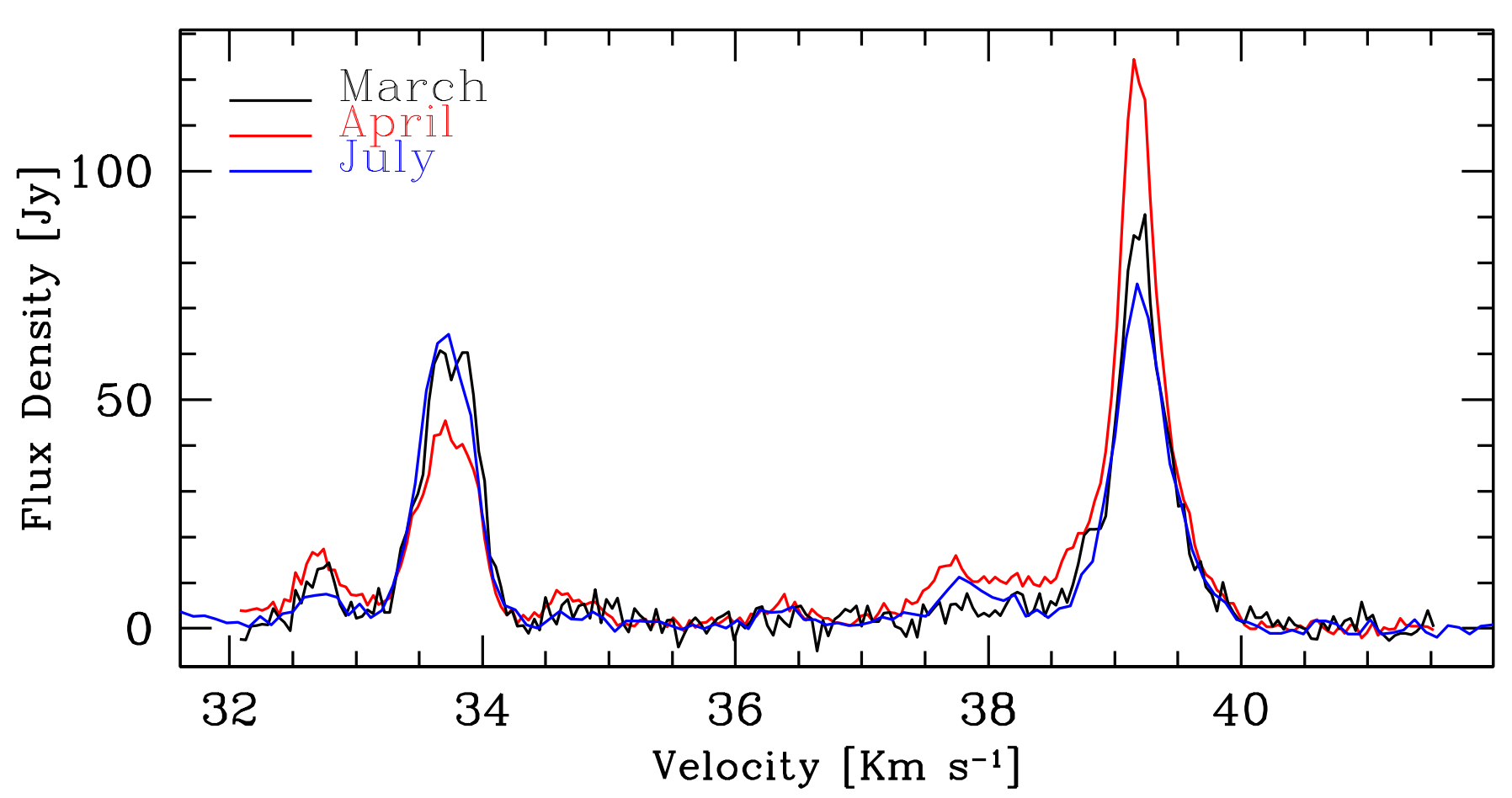

Fig. 1. 6.7 GHz methanol maser spectra of the three epochs, obtained by summing all the pixels in the image for each channel.
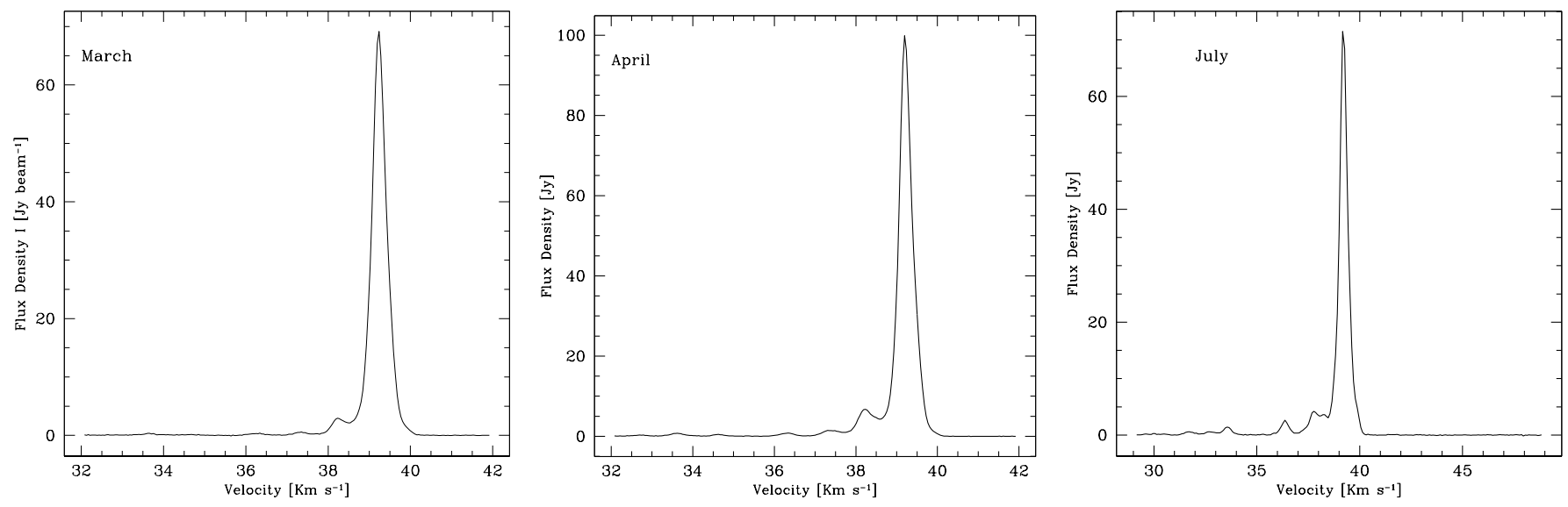

Fig. 2. Spectra of the brightest maser feature F.01 in the three epochs.

The values for $P_{1}$ and $\chi$ are also reported in Tables 3 and 4 . In our polarisation analysis we only considered maser features with intensity $>1 \mathrm{Jy}$.

All the masers that we identified are plotted in Fig. 3. The bottom panels show a zoom of the regions marked by the dashed grey boxes in the top panels, to better discern between very close maser features. Each maser is represented by a triangle. The different sizes of the triangles represent the intensity, while the colours indicate the velocity of the maser feature, following the scale reported in the colour bar. For March and April only, the line segments mark the direction of the polarisation angle for the maser features that show linear polarisation. Under the assumption that the angle between the magnetic field and the line of sight is $\theta>\theta_{\text {crit }} \sim 55^{\circ}$, where $\theta_{\text {crit }}$ is the Van Vleck angle (e.g. Surcis et al. 2011), we considered the linear polarisation perpendicular to the magnetic field. The vectors are also scaled logarithmically according to $P_{1}$ as reported in Tables 3 and 4 . We also plot, in the bottom right corners, the average direction of the resulting magnetic field $\Phi_{B}$ obtained for two groups of masers as defined in Sect. 5.2. For the F.06 feature we tentatively detect a circular polarisation signature and the magnetic field along the line of sight (see Sect. 5.4).

\section{Discussion}

\subsection{Maser distribution and kinematics}

Goedhart et al. (2009) presented a spectrum of the $6.7 \mathrm{GHz}$ methanol maser emission of IRAS 18089. The spectrum is divided in two distinct blocks around two main peaks: one located at a velocity of $33.7 \mathrm{~km} \mathrm{~s}^{-1}$ and another at $39.2 \mathrm{~km} \mathrm{~s}^{-1}$. In our observations, we found a similar spectrum, with all our maser features grouped around those two values (see Tables 3-5): as shown in the tables, the velocity shifts are within the channel width, apart from F.06 which presents a shift of $\sim 0.2 \mathrm{~km} \mathrm{~s}^{-1}$, 
D. Dall'Olio et al.: Magnetic field in IRAS 18089

Table 3. Parameters of the 6.7-GHz methanol maser features detected in IRAS 18089-1732 in March.

\begin{tabular}{|c|c|c|c|c|c|c|c|c|c|}
\hline $\begin{array}{c}(1) \\
\text { Maser }\end{array}$ & $\begin{array}{l}\text { (2) } \\
\text { RA }^{a} \\
\text { offset } \\
\text { (mas) }\end{array}$ & $\begin{array}{l}(3) \\
\text { Dec }^{a} \\
\text { offset } \\
\text { (mas) }\end{array}$ & $\begin{array}{c}(4) \\
\text { Peak flux } \\
\text { density }(I) \\
\left(\text { Jy beam }^{-1}\right)\end{array}$ & $\begin{array}{c}(5) \\
V_{\mathrm{lsr}} \\
\left(\mathrm{km} \mathrm{s}^{-1}\right)\end{array}$ & $\begin{array}{l}(6) \\
P_{1}^{b} \\
(\%)\end{array}$ & $\begin{array}{l}(7) \\
\chi^{b} \\
\left({ }^{\circ}\right)\end{array}$ & $\begin{array}{c}(8) \\
\Delta_{V_{L}} \\
\left(\mathrm{~km} \mathrm{~s}^{-1}\right)\end{array}$ & $\begin{array}{l}(9) \\
P_{\mathrm{V}} \\
(\%)\end{array}$ & $\begin{array}{l}(10) \\
B_{\mathrm{los}} \\
(\mathrm{mG})\end{array}$ \\
\hline F.01 & $0 \pm 0.13$ & $0 \pm 0.78$ & $69.99 \pm 0.66$ & 39.24 & $8.9 \pm 1.4$ & $-78 \pm 5$ & - & - & - \\
\hline F.02 & $-34.90 \pm 0.21$ & $14.11 \pm 1.26$ & $8.53 \pm 0.13$ & 38.84 & - & - & - & - & - \\
\hline F.03 & $43.68 \pm 0.15$ & $-2.46 \pm 0.88$ & $5.19 \pm 0.06$ & 37.75 & - & & - & - & - \\
\hline F.04 & $28.92 \pm 0.18$ & $15.60 \pm 1.11$ & $3.23 \pm 0.04$ & 36.43 & - & - & - & - & - \\
\hline F.05 & $157.26 \pm 0.16$ & $51.95 \pm 0.96$ & $4.12 \pm 0.05$ & 34.67 & - & - & - & - & - \\
\hline F.06 & $1098.47 \pm 1.07$ & $1128.63 \pm 6.43$ & $40.04 \pm 0.40$ & 33.84 & $3.8 \pm 2.7$ & $-50 \pm 28$ & 0.4 & 0.8 & $5.5 \pm 1.7$ \\
\hline F.07 & $54.65 \pm 0.21$ & $54.52 \pm 1.28$ & $17.10 \pm 0.27$ & 33.53 & $6.3 \pm 0.4$ & $-31 \pm 1$ & - & - & - \\
\hline F.08 & $55.04 \pm 0.15$ & $44.99 \pm 0.91$ & $6.45 \pm 0.07$ & 32.74 & $9.4 \pm 0.3$ & $-16 \pm 1$ & - & - & - \\
\hline F.09 & $937.80 \pm 0.16$ & $1620.90 \pm 0.94$ & $3.45 \pm 0.07$ & 32.70 & - & - & - & - & - \\
\hline
\end{tabular}

Notes. ${ }^{(a)}$ The offsets are relative to the position of F.01 in March, i.e. $\alpha_{2000}=18^{\mathrm{h}} 11^{\mathrm{m}} 51.3954^{\mathrm{s}} \pm 0.0001^{\mathrm{s}}, \delta_{2000}=-17^{\circ} 31^{\prime} 29.92^{\prime \prime} \pm 0.01^{\prime \prime} .{ }^{(b)} P_{1}$ and $\chi$ are the median values of the linear polarisation fraction and the linear polarisation angle measured across the spectrum, respectively.

Table 4. Parameters of the 6.7-GHz methanol maser features detected in IRAS 18089-1732 in April.

\begin{tabular}{|c|c|c|c|c|c|c|c|c|c|}
\hline $\begin{array}{c}(1) \\
\text { Maser }\end{array}$ & $\begin{array}{l}(2) \\
\mathrm{RA}^{a} \\
\text { offset } \\
\text { (mas) }\end{array}$ & $\begin{array}{l}(3) \\
\text { Dec }^{a} \\
\text { offset } \\
\text { (mas) }\end{array}$ & $\begin{array}{c}(4) \\
\text { Peak flux } \\
\text { density }(I) \\
\left(\mathrm{Jy} \mathrm{beam}^{-1}\right)\end{array}$ & $\begin{array}{c}(5) \\
V_{\mathrm{lsr}} \\
\left(\mathrm{km} \mathrm{s}^{-1}\right)\end{array}$ & $\begin{array}{l}(6) \\
P_{1}^{b} \\
(\%)\end{array}$ & $\begin{array}{l}(7) \\
\chi^{b} \\
\text { (o) }\end{array}$ & $\begin{array}{c}(8) \\
\Delta_{V_{L}} \\
\left(\mathrm{~km} \mathrm{~s}^{-1}\right)\end{array}$ & $\begin{array}{l}(9) \\
P_{\mathrm{V}} \\
(\%)\end{array}$ & $\begin{array}{c}(10) \\
B_{\mathrm{los}} \\
(\mathrm{mG})\end{array}$ \\
\hline F.01 & $0 \pm 0.10$ & $0 \pm 0.59$ & $122.29 \pm 0.89$ & 39.20 & $8.5 \pm 1.3$ & $-72 \pm 5$ & - & - & - \\
\hline F.02 & $-36.16 \pm 0.14$ & $24.21 \pm 0.85$ & $13.38 \pm 0.14$ & 38.76 & - & - & - & - & - \\
\hline F.10 & $0 \pm 0.11$ & $-0.18 \pm 0.68$ & $8.39 \pm 0.07$ & 38.23 & $6.9 \pm 0.7$ & $-68 \pm 7$ & - & - & - \\
\hline F.03 & $48.23 \pm 0.09$ & $18.08 \pm 0.55$ & $17.57 \pm 0.12$ & 37.70 & - & - & - & - & - \\
\hline F.04 & $30.23 \pm 0.10$ & $24.10 \pm 0.60$ & $6.71 \pm 0.05$ & 36.39 & $9.4 \pm 1.2$ & $-16 \pm 1$ & - & - & - \\
\hline F.05 & $162.97 \pm 0.08$ & $72.11 \pm 0.51$ & $11.22 \pm 0.07$ & 34.67 & $4.0 \pm 0.6$ & $-46 \pm 1$ & - & - & - \\
\hline F.06 & $1110.26 \pm 0.15$ & $1127.96 \pm 0.87$ & $42.78 \pm 0.46$ & 33.66 & $6.4 \pm 2.6$ & $-16 \pm 12$ & 0.4 & 0.3 & $4.9 \pm 1.5$ \\
\hline F.07 & $60.30 \pm 0.13$ & $72.19 \pm 0.81$ & $39.11 \pm 0.39$ & 33.58 & $8.8 \pm 0.1$ & $-32 \pm 1$ & - & - & - \\
\hline F.09 & $941.32 \pm 0.41$ & $1632.09 \pm 2.47$ & $3.95 \pm 0.12$ & 32.70 & - & - & - & - & - \\
\hline
\end{tabular}

Notes. ${ }^{(a)}$ The offsets are relative to the position of F.01 in April, i.e. $\alpha_{2000}=18^{\mathrm{h}} 11^{\mathrm{m}} 51.3929^{\mathrm{s}} \pm 0.0003^{\mathrm{s}}, \delta_{2000}=-17^{\circ} 31^{\prime} 29.86^{\prime \prime} \pm 0.03^{\prime \prime} .^{(b)} P_{1}$ and $\chi$ are the median values of the linear polarisation fraction and the linear polarisation angle measured across the spectrum, respectively.

Table 5. Parameters of the 6.7-GHz methanol maser features detected in IRAS 18089-1732 in July.

\begin{tabular}{ccccc}
\hline \hline $\begin{array}{c}(1) \\
\text { Maser }\end{array}$ & $\begin{array}{c}(2) \\
\text { RA }^{a} \\
\text { offset } \\
(\mathrm{mas})\end{array}$ & $\begin{array}{c}(3) \\
\mathrm{Dec}^{a} \\
\text { offset } \\
(\mathrm{mas})\end{array}$ & $\begin{array}{c}(4) \\
\text { Peak flux } \\
\text { density }(I) \\
\left(\text { Jy beam }^{-1}\right)\end{array}$ & $\begin{array}{c}(5) \\
V_{\mathrm{lsr}} \\
\left(\mathrm{km} \mathrm{s}^{-1}\right)\end{array}$ \\
\hline F.01 & $0 \pm 0.01$ & $0 \pm 0.07$ & $74.89 \pm 0.06$ & 39.18 \\
F.03 & $45.05 \pm 0.23$ & $-1.50 \pm 1.37$ & $10.06 \pm 0.17$ & 37.77 \\
F.05 & $156.27 \pm 0.28$ & $53.67 \pm 1.65$ & $3.77 \pm 0.08$ & 34.70 \\
F.06 & $1104.28 \pm 0.03$ & $1123.61 \pm 0.17$ & $47.90 \pm 0.10$ & 33.73 \\
F.07 & $56.79 \pm 0.08$ & $60.12 \pm 0.49$ & $18.42 \pm 0.11$ & 33.56 \\
F.09 & $938.19 \pm 0.50$ & $1620.49 \pm 3.00$ & $3.25 \pm 0.12$ & 32.68 \\
F.11 & $59.70 \pm 0.32$ & $35.35 \pm 1.94$ & $2.47 \pm 0.06$ & 30.05 \\
\hline
\end{tabular}

Notes. ${ }^{(a)}$ The offsets are relative to the position of F.01 in July, i.e. $\alpha_{2000}=18^{\mathrm{h}} 11^{\mathrm{m}} 51.399^{\mathrm{s}} \pm 0.002^{\mathrm{s}}, \delta_{2000}=-17^{\circ} 31^{\prime} 29.93^{\prime \prime} \pm 0.1^{\prime \prime}$.

probably due to a blend of two components (see Sect. 5.4). Since the same velocities occur in all three of our epochs, we divided the masers in two groups: a blue group spanning a velocity range from 30.0 to $36.4 \mathrm{~km} \mathrm{~s}^{-1}$ (containing F.04, F.05, F.06, F.07, F.08, F.09, and F.11), and a red group from 37.7 to
$39.2 \mathrm{~km} \mathrm{~s}^{-1}$ (containing F.01, F.02, F.03, and F.10). The blue group presents a velocity that is similar to the $v_{\mathrm{lsr}}$ of the compact core ( $\sim 33.8 \mathrm{~km} \mathrm{~s}^{-1}$, Beuther et al. 2005; Leurini et al. 2007) while the red group is closer to the velocity of the $\mathrm{CO}$ outflow ( $\sim 40 \mathrm{~km} \mathrm{~s}^{-1}$, Beuther et al. 2010). 

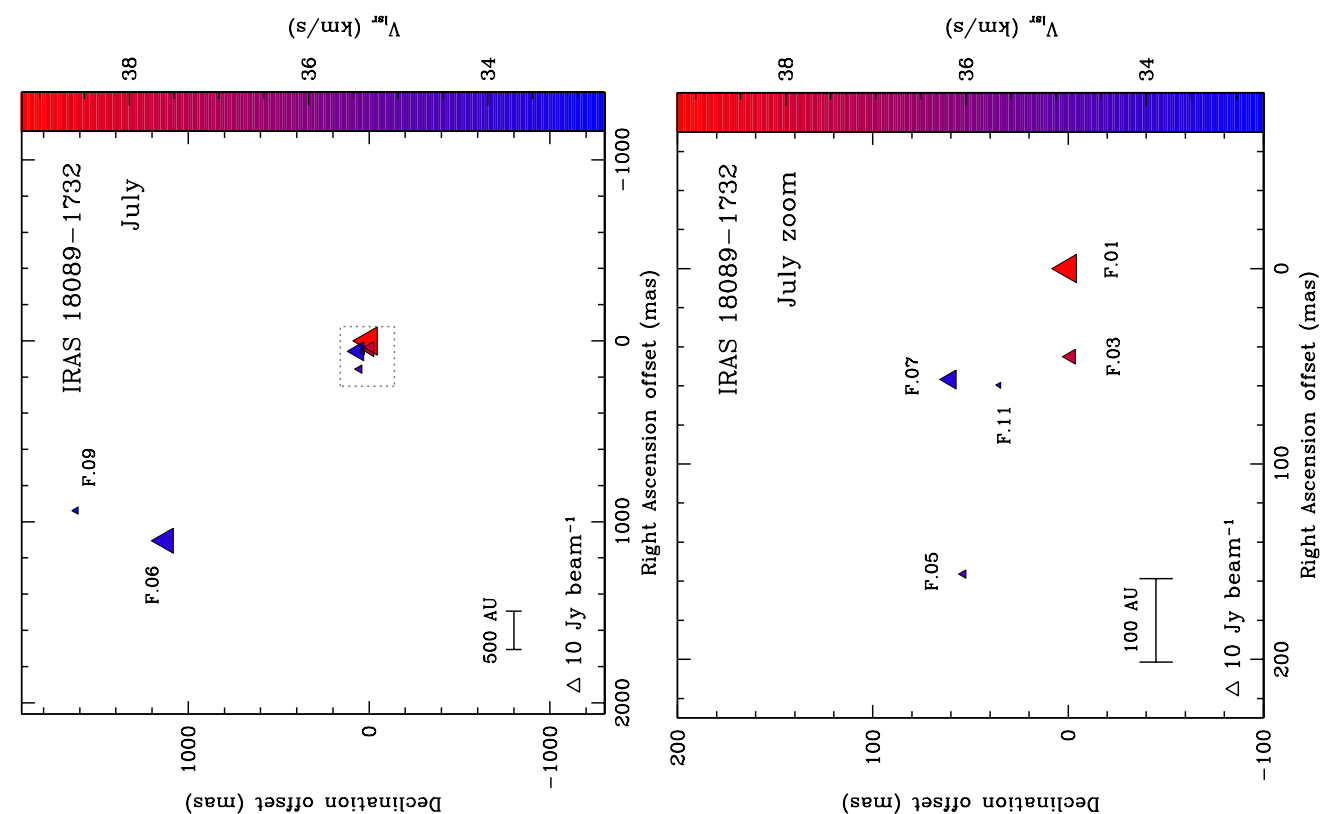

$\dot{2}$

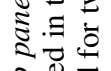

s

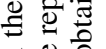

플

응

원

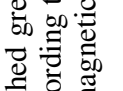

$(\mathrm{s} / \mathrm{wy})^{\mathrm{sen} \Lambda}$

(s/Ur) ${ }^{4 s 1} \Lambda$

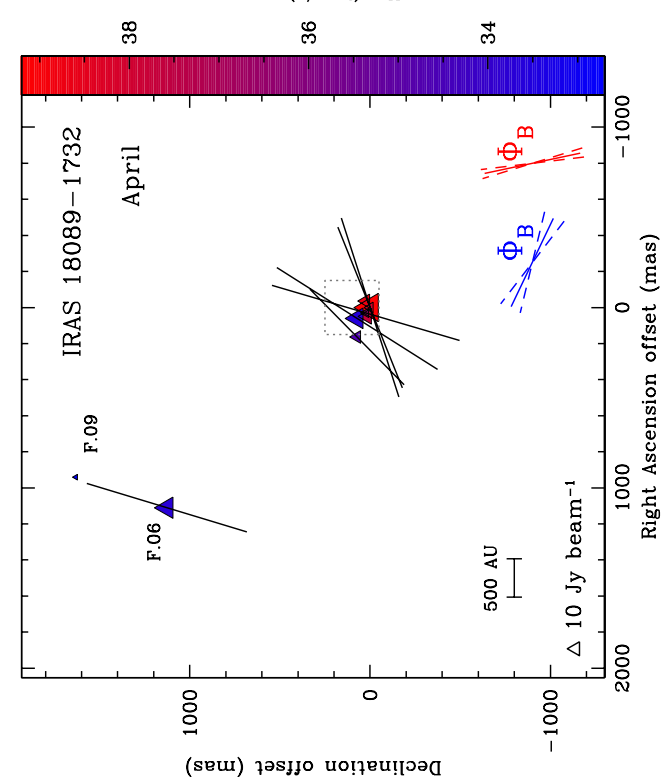

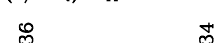

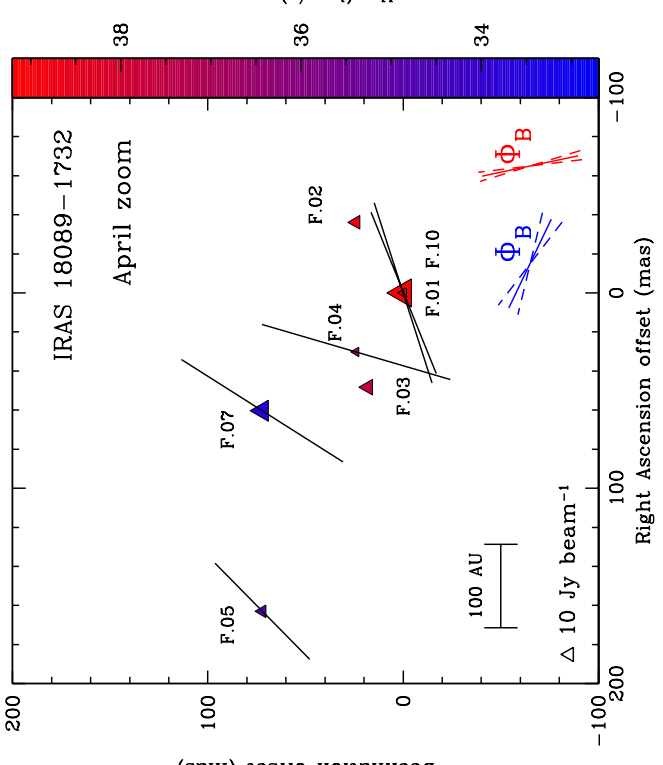

尊宕

我目

召吾焉

矛志.

호월

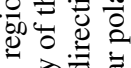

氜昰

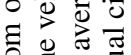

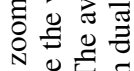

๙

诺

s.

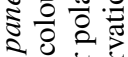

ఏ

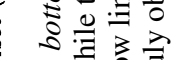

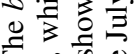

in 窟

के चे क्ष

क. 过离

西焉

$\Xi$ 苛

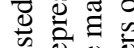

品

$\Xi$

(s/ur) ${ }^{\mathrm{sel}} \Lambda$

(s/Uy) ${ }^{\text {sel } \Lambda}$

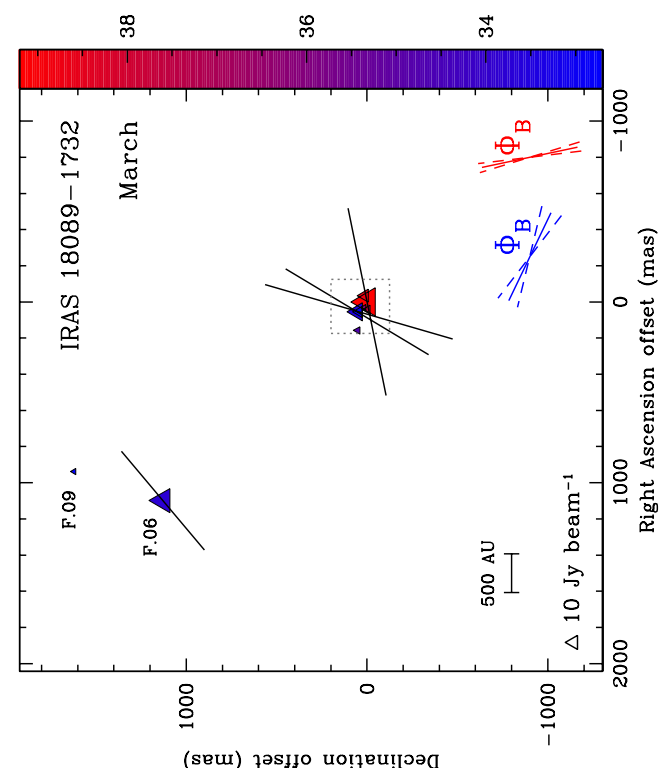

$\infty$

\pm
$>$

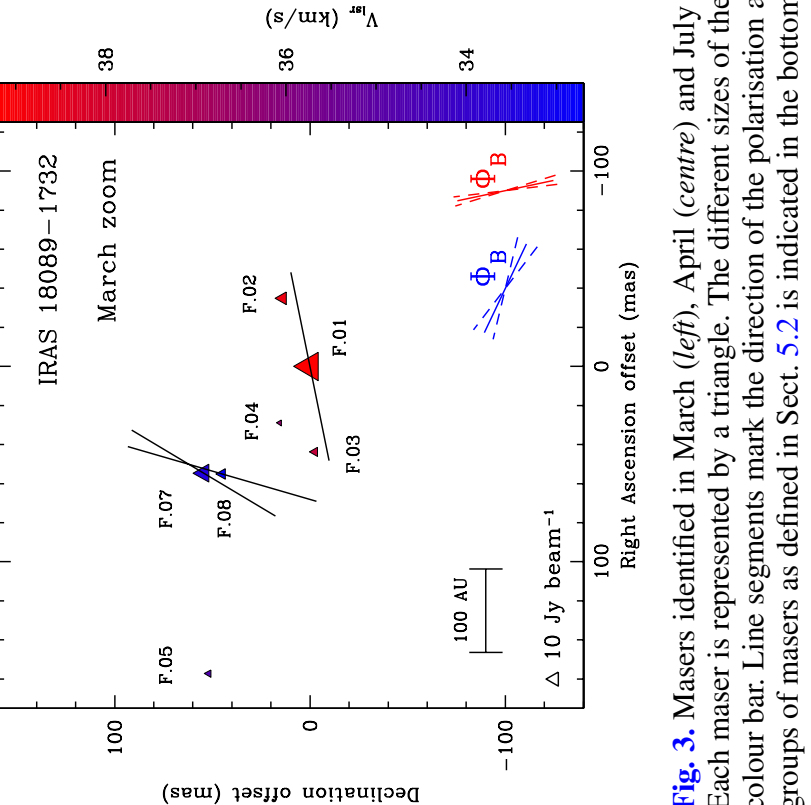

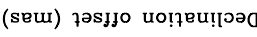

부ㅇㅝㅠ 

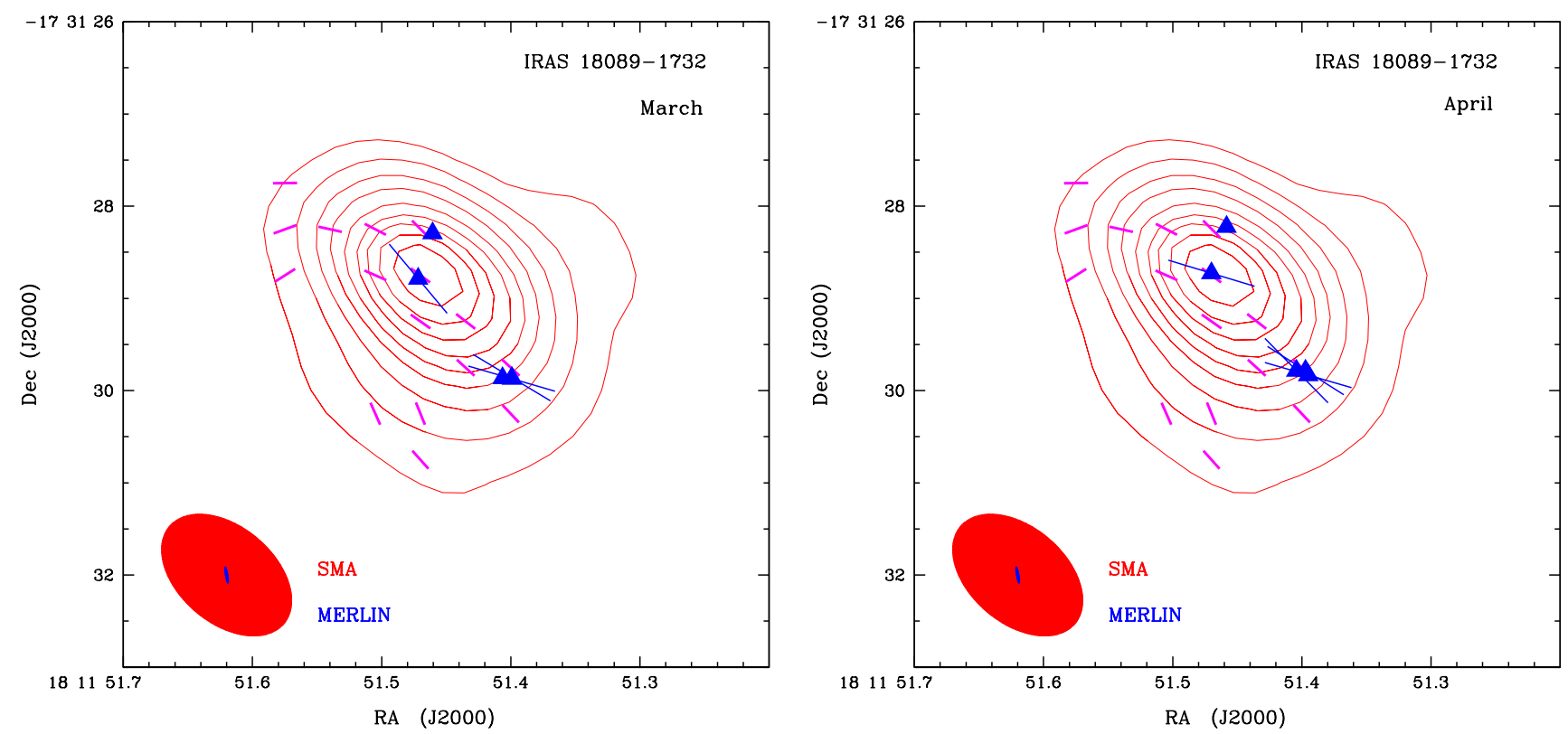

Fig. 4. Masers in the blue group (blue triangles and blue segments) superimposed on the integrated I image of the dust continuum emission observed by Beuther et al. (2010) at $880 \mu \mathrm{m}$ with SMA (red contours; the contours are drawn in $10 \sigma$ steps). The magenta line segments show the magnetic field orientation obtained by linearly polarised dust emission (Beuther et al. 2010). The blue segments represent the magnetic field orientation obtained by our linearly polarised methanol maser emission (Sect. 5.2); therefore the magnetic field follows the same direction indicated by the dust emission. The red and blue ellipses show the beams of SMA $\left(1.65^{\prime \prime} \times 1.05^{\prime \prime}\right.$, position angle $\left.51^{\circ}\right)$ and MERLIN, respectively. Left panel: March; right panel: April.

\subsection{Orientation of the magnetic field}

The difference in velocity and the separation of the two groups is also preserved in the orientation of the polarisation vector of each maser in both March and April. The two groups of masers show ordered linear polarisation vectors. The blue (B) group has a weighted average angle of $\left\langle\chi_{\mathrm{B}, \mathrm{M}}\right\rangle=-24^{\circ} \pm 8^{\circ}$ in March $(\mathrm{M})$ and $\left\langle\chi_{\mathrm{B}, \mathrm{A}}\right\rangle=-31^{\circ} \pm 12^{\circ}$ in April (A). The red (R) group has only one maser feature presenting linear polarisation in March with an angle $\left\langle\chi_{\mathrm{R}, \mathrm{M}}\right\rangle=-78^{\circ} \pm 5^{\circ}$, while in April the weighted polarisation angle is $\left\langle\chi_{\mathrm{R}, \mathrm{A}}\right\rangle=-70^{\circ} \pm 2^{\circ}$. Therefore the two groups show distinct polarisation angles as well as velocities.

The presence of these two groups of masers, showing two characteristic orientations of the polarisation angle and two velocity ranges, can be interpreted as a distinct signature of masers originating in two different regions around the protostar. Under this hypothesis, the two groups of masers probe the magnetic field morphology in two different regions. In Fig. 3 we plot the two directions of the resulting magnetic field $\Phi_{B}$, assuming a magnetic field perpendicular to the polarisation angles (see Sect. 4). The masers in the blue group have an orientation on the plane of the sky $\Phi_{B}^{\text {disc }}=+62^{\circ} \pm 3^{\circ}$, probing the magnetic field close to the disc. Conversely, the masers in the red group have an orientation on the plane of the sky $\Phi_{B}^{\text {outflow }}=+14^{\circ} \pm 4^{\circ}$, probing the magnetic field close to the base of the outflow.

Beuther et al. (2010) detected dust polarised emission describing the IRAS 18089 disc, and the accompanying line features have a velocity similar to that identified for the blue group of masers. In Fig. 4 we overplot the blue group masers on the Stokes $I$ continuum emission observed by Beuther et al. (2010). The red and blue ellipses represent the SMA and MERLIN beams, respectively. In the left panel we plot the masers identified in March, while in the right panel those identified in April. The blue lines show the direction of the magnetic field, obtained by our maser linear polarisation analysis. The magenta segments identify the magnetic field direction as found by Beuther et al. (2010) from the polarised dust emission. This suggests that the small-scale magnetic field probed by the masers is consistent with the large-scale magnetic field traced by the dust. Therefore we can conclude that the magnetic field structures remain consistent over many orders of magnitude in scales.

Beuther et al. (2010) also found $\mathrm{CO}(3-2)$ emission tracing the outflow at a velocity consistent with that found for our red group masers. However, the $\mathrm{CO}(3-2)$ outflow is located too far away $\left(\sim 4^{\prime \prime}\right.$ from the peak of $\mathrm{CO}(3-2)$ and $\sim 1.5^{\prime \prime}$ from the lowest contour) from the red group masers, so it is not tracing the same gas. Beuther et al. (2004b) observed several other molecules tracing the outflow including $\mathrm{SiO}(5-4)$ and $\mathrm{H}_{2} \mathrm{~S}$. Both $\mathrm{SiO}(5-4)$ and $\mathrm{H}_{2} \mathrm{~S}$ present a more extended emission than that of $\mathrm{CO}(3-2)$, that overlaps with the area where our red group masers lie. The channel maps present by Beuther et al. (2004a,b) show that both $\mathrm{SiO}(5-4)$ and $\mathrm{H}_{2} \mathrm{~S}$ have a velocity consistent with our red group masers. Our hypothesis is that the red group masers are tracing the magnetic field responsible for, or related to, the outflow.

\subsection{Maser variability}

Goedhart et al. (2009) observed a quasi-periodic variability in IRAS 18089. During 9 years of observations they registered a light curve presenting regular minima but with variable amplitude of flares and phase of the peak. The most likely period is $29.5 \pm 0.1$ days. The feature at $39.2 \mathrm{~km} \mathrm{~s}^{-1}$ takes roughly $12 \mathrm{~d}$ on average to reach the maximum after a minimum. Extrapolating from the last minimum observed by Goedhart et al. (2009) in 2007, we can predict the dates of the maxima and minima for the features in common with our observations (see Table 6 and Fig. 5). The uncertainties reported in Table 6 have been calculated by multiplying the uncertainty on the period $(0.1 \mathrm{~d})$ by the square root of the number of elapsed periods between the 

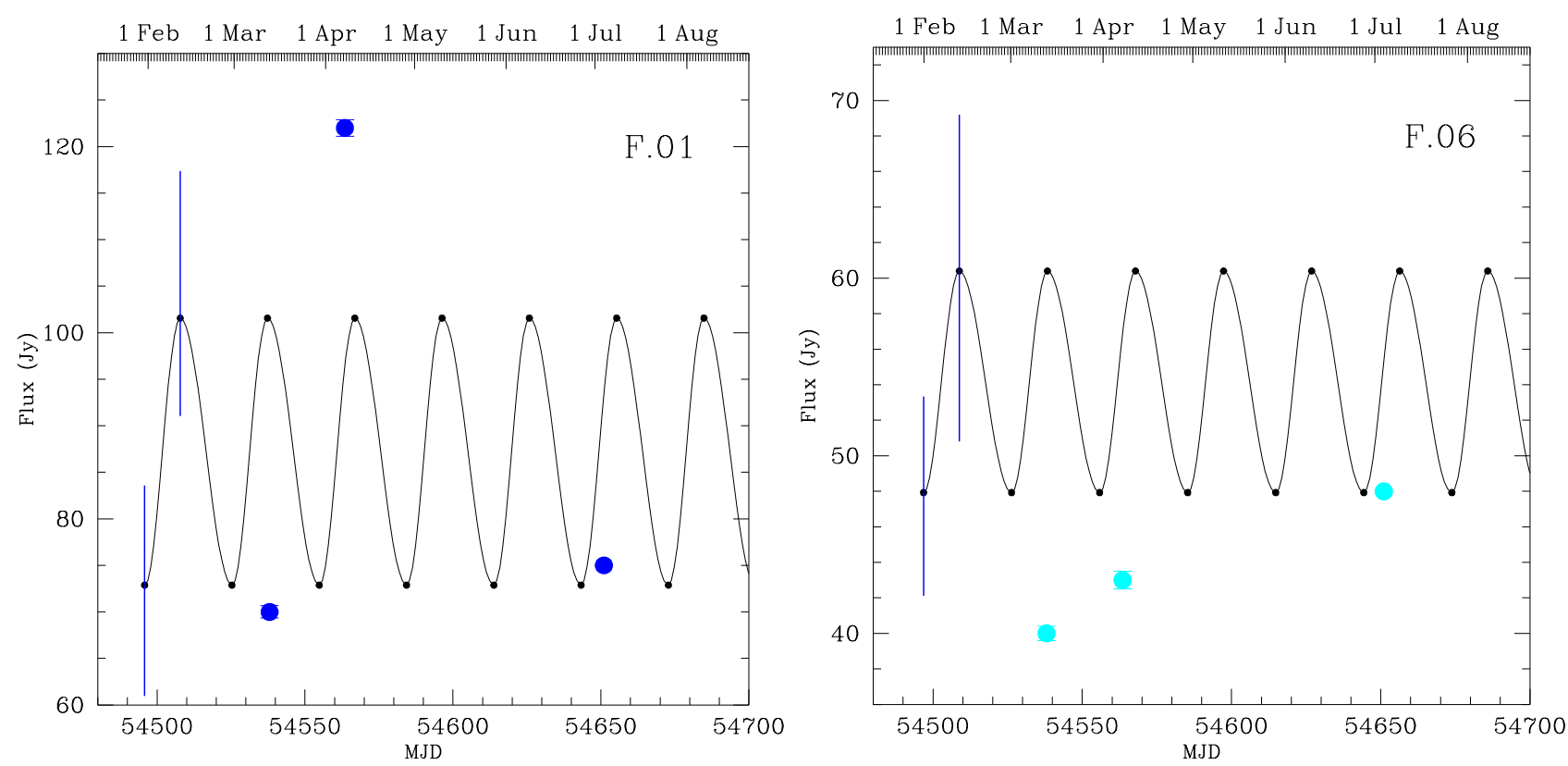

Fig. 5. Flux density versus time for the features F.01 (left) and F.06 (right). The black curve illustrates the expected variability of the maser feature according to Goedhart et al. (2009); the predicted times of maxima and minima are reported in Table 6 . The blue bars indicate the range of the intensities between the highest maximum and the lowest maximum and between the highest minimum and lowest minimum.

Table 6. Predicted days and $\mathrm{h}$ of minima and maxima in the light curve of the $39.2 \mathrm{~km} \mathrm{~s}^{-1}$ feature F.01, extrapolated from the light curve in Goedhart et al. (2009).

\begin{tabular}{ccccc}
\hline \hline \multicolumn{2}{c}{$\begin{array}{c}\text { Expected } \\
\text { minima }\end{array}$} & \multicolumn{2}{c}{$\begin{array}{c}\text { Expected } \\
\text { maxima }\end{array}$} & $\begin{array}{c}\text { Uncertainty } \\
\text { (days) }\end{array}$ \\
\hline 29-02-2008 & $07: 30$ & $12-03-2008$ & $07: 30$ & \pm 0.3 \\
29-03-2008 & $19: 30$ & $10-04-2008$ & $19: 30$ & \pm 0.3 \\
28-04-2008 & $07: 30$ & $10-05-2008$ & $07: 30$ & \pm 0.4 \\
27-05-2008 & $19: 30$ & $08-06-2008$ & $19: 30$ & \pm 0.4 \\
26-06-2008 & $07: 30$ & $08-07-2008$ & $07: 30$ & \pm 0.4 \\
25-07-2008 & $19: 30$ & $06-08-2008$ & $19: 30$ & \pm 0.4 \\
\hline
\end{tabular}

Notes. The predicted minima and maxima for F.02 can be obtained adding one day to the dates in the table.

last minimum observed by Goedhart et al. (2009) and our observations (12-16 periods). Our March observations took place one day after a predicted maximum of F.01, whilst the April and July observations took place 2-3 and 3-4 days beforehand, respectively.

In Fig. 1, the peaks around 33.8 and $39.2 \mathrm{~km} \mathrm{~s}^{-1}$ are dominated by F.06 and F.01, respectively, which from this plot and Tables 3-5 can be seen to vary strongly in peak intensity. As indicated in Fig. 5, left panel, F.01 is brightest in April, at $\sim 122 \mathrm{Jy} \mathrm{beam}^{-1}$, close to a predicted peak, but the March and July observations, although also close to predicted peaks, have flux densities of 70-75 $\mathrm{Jy}^{\text {beam }^{-1}}$, closer to the values at minimum reported by Goedhart et al. (2009). They found that the period of F.06 lags F.01 by 1 day, so we would also expect to see F.06 close to maximum. From Fig. 5, right panel, we instead measured flux densities of 40-47 $\mathrm{Jy} \mathrm{beam}^{-1}$ which are closer to the minimum values found by Goedhart et al. (2009). This is not entirely unexpected, since Goedhart et al. (2009) pointed out that the times of maxima are not very regular. Another possibility is that these features have dropped in flux density (since we did not observe at the predicted minima, it is possible that the features had much lower fluxes then).
Following Goedhart et al. (2005, 2009), a possible way to understand the delay between the peaks is by analysing the projected distances of the masers and the light travel times. The projected distance between F.01 and F.06 is 3700 au and the light travel time across this length is $\sim 20 \mathrm{~d}$. Goedhart et al. (2009) observed delays also for other features. Between F.01 and F.02 and F.01 and F.03 the expected delays are -1.7 and -3.3 days, respectively. The projected distance between F.01 and F.02 features is $\sim 90$ au and the corresponding light travel time is $\sim 0.5 \mathrm{~d}$. In the case of F.01 and F.03 the projected distance is $100 \mathrm{au}$, equivalent to $\sim 0.6$ light days. Between F.01 and F.05 the observed delay is $1.7 \mathrm{~d}$, and the projected distance is $\sim 380$ au corresponding to $\sim 2.3$ light days. The features with a negative delay coincide with our red group, while the features with positive delays coincide with the blue group. If we assume that the variability of all the features is due to the same pumping source, it should be located on a plane between F.01 and F.06. F.02 and F.03 should be located closer to the pumping source than F.01. However, because of the degeneracy of the positions along the line of sight, many configurations are possible and at the current status we cannot determine a single three-dimensional model of the region.

Walsh et al. (1998) provided a map of IRAS 18089 masers and obtained relative positions with an accuracy of $\sim 0.05^{\prime \prime}$ and absolute positions accurate to $\sim 1^{\prime \prime}$. Through the study of position-velocity $(P-V)$ diagrams, no evidence of peculiar structure within the maser site was found. We compared the relative positions of the masers observed by Walsh et al. (1998) assuming the same absolute position for the brightest features at $39.2 \mathrm{~km} \mathrm{~s}^{-1}$. For an easy comparison, we report, in Table 8, the maser features observed in our MERLIN observations, in Walsh et al. (1998), and in Goedhart et al. (2009). In our three epochs we observed almost all the features already detected by Walsh et al. (1998), at positions within their reported uncertainty of 50 mas. However, there are some exceptions: our F.04, F.07, F.08, F.09 and F.10 were not detected by Walsh et al. (1998), while we did not detect features D, G, and H at 32.7 and $31.6 \mathrm{~km} \mathrm{~s}^{-1}$. 

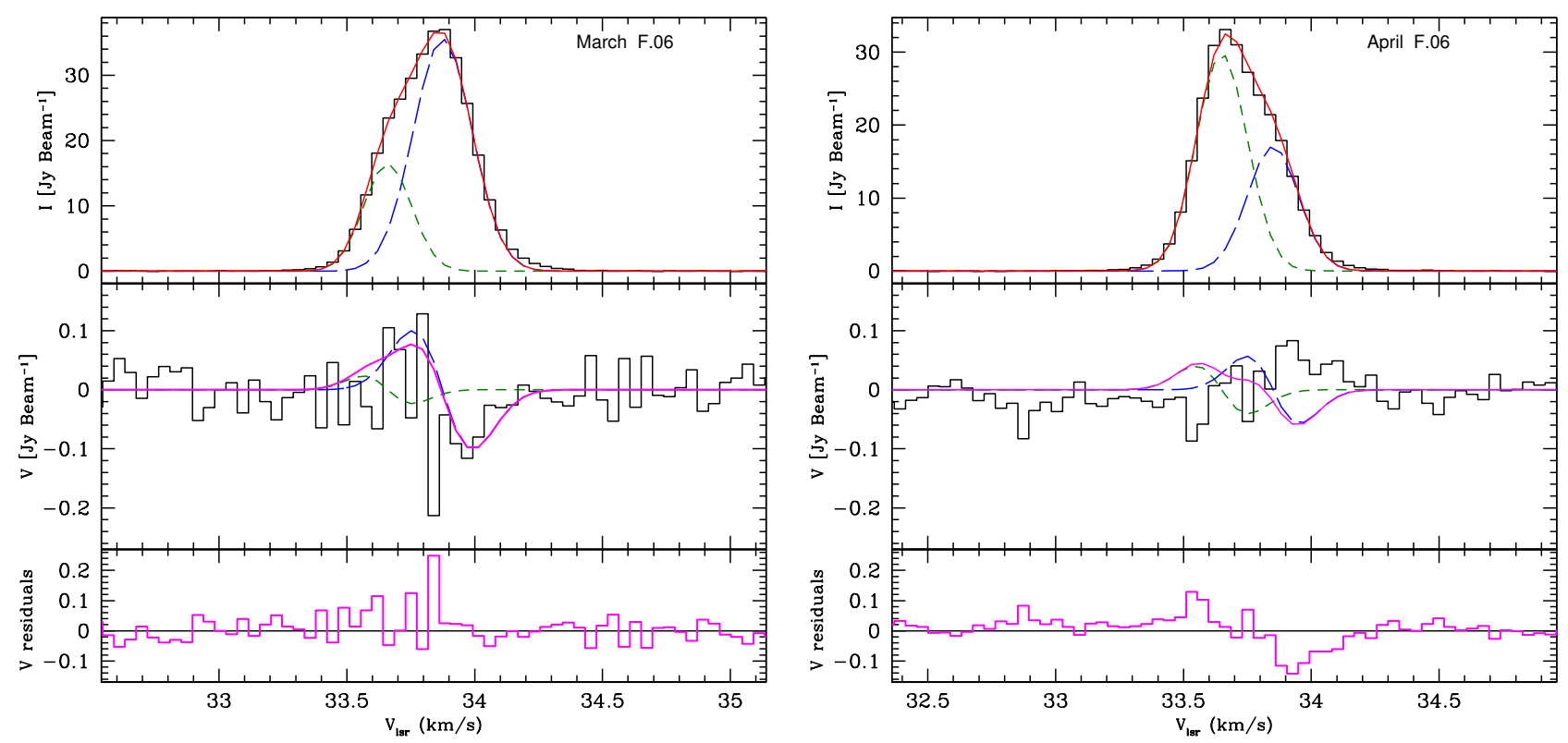

Fig. 6. Fit to maser feature F.06 assuming the presence of two hyperfine components; left: March observation, right: April observation. Upper panels: total intensity $I$ spectrum plotted as the black histogram. The solid red curve in the upper panel is the best fit using two Gaussian components, representing two maser hyperfine components separated by $0.2 \mathrm{~km} \mathrm{~s}^{-1}$ (see Sect. 5.4). Component 1 is the blue long-dashed line and component 2 is the green short-dashed line. Middle panels: circular polarisation $V$ spectrum (black histogram). The solid magenta line is the sum of the derivatives of the two components of the fit from the upper panel. The blue long-dashed line is the derivative of component 1 and the green short-dashed line is the derivative of component 2. Lower panels: we plot the residuals between the circular polarisation (middle panel, black histogram) and the sum of the derivatives (middle panel, solid magenta line).

\subsection{Strength of the magnetic field}

The strength of the magnetic field along the line of sight can be obtained from the circular polarisation (Vlemmings et al. 2001, 2006; Surcis et al. 2014a). Previous works showed that the circular polarisation fraction in methanol masers is typically very weak ( $<1 \%$; e.g. Surcis et al. 2015), so circular polarisation can be observed more easily in the brightest maser features. However, as shown by Vlemmings et al. (2001, 2002), an increase of the noise, a narrowing and re-broadening, or a change in shape of the maser line can also occur in hyperfine interactions or in 3D maser propagation effect. Therefore, as suggested by Surcis et al. (2015), a detection of circular polarisation should only be considered real if it presents a $V$ peak flux density at least five times higher than the rms.

In our observations, we found several of the above effects that made it difficult to identify the Zeeman effect. Therefore, we only propose a tentative detection of circular polarisation for one maser feature (reported as F.06 in Tables 3 and 4, respectively) shown in Fig. 6. For F.06 we tentatively compute the magnetic field strength along the line of sight.

The observed $V$ spectrum is a sin-shaped function, corresponding to the derivative $I^{\prime}$ of the total power spectrum $I$ (Troland \& Heiles 1982). By fitting Gaussian components to the $I$ spectrum, and the corresponding derivative to the $V$ spectrum, we can take

$V=a I+b \frac{\mathrm{d} I}{\mathrm{~d} v}$

where $a$ and $b=z B \cos \theta$ are fit parameters, together with the Gaussian components' intensity, centre velocity, and line width. $B$ is the magnetic field strength, $\theta$ is the angle between the magnetic field and the line of sight and $z$ is the Zeeman splitting factor for $\mathrm{CH}_{3} \mathrm{OH}$. However, $z$ depends on the Landé $g$-factor, which was unknown for the methanol maser molecules until recently. Therefore, all the previous estimation of $B$ along the line of sight $\left(B_{\text {los }}\right)$ were affected by this uncertainty. Recently a list of $z$ factor values has been estimated for the $6.7 \mathrm{GHz}$ methanol transition $5_{15} \mathrm{~A}_{2} \leftarrow 6_{06} \mathrm{~A}_{1}$ and all its possible hyperfine components (Lankhaar et al. 2017).

In Fig. 6, we show the Stokes I profile (upper panels, black histogram) for the maser feature F.06, in March (left panel) and April (right panel). We tried to fit the line using two Gaussian components separated by $\sim 0.2 \mathrm{~km} \mathrm{~s}^{-1}$, and we plot the best fit in red, component 1 is the blue long-dashed line and component 2 is the green short-dashed line. We summarise the best fit parameters in Table 9.

It is possible to see that the $I$ spectrum in April presents a reversed profile with respect to March. A similar behaviour is also seen in the circular polarisation spectra $V$, plotted as black histograms in the middle panels of Fig. 6: it presents two S-shape profiles, one being the opposite of the other.

Following Lankhaar et al., a possible explanation of such $I$ profiles and opposite circular polarisation could be due to the presence of two hyperfine components of the $6.7 \mathrm{GHz}$ methanol transition $5_{15} \mathrm{~A}_{2} \rightarrow 6_{06} \mathrm{~A}_{1}$ : the $F=3 \rightarrow 4$ (component 1 ) and the $F=4 \rightarrow 5$ (component 2), also separated by $\sim 0.2 \mathrm{~km} \mathrm{~s}^{-1}$ (Lankhaar et al. 2016). Under this hypothesis, one hyperfine transition would be preferred over the other in one epoch, and vice versa in the following epoch. Therefore, we tried to fit our $V$ spectra using the sum of the derivatives of the two hyperfine components (magenta line in the middle panels of Fig. 6). The derivatives of the single components are also plotted: the derivative of component 1 is the blue long-dashed line and the derivative of component 2 is the green short-dashed line. The March $V$ spectrum can be reproduced (Fig. 6, left middle panel) by using two hyperfine components having a Zeeman coefficient $z$ of $-1.135 \mathrm{~Hz} \mathrm{mG}{ }^{-1}$ for component 1 and $-0.467 \mathrm{~Hz} \mathrm{mG}^{-1}$ for component 2: the resulting $B_{\text {los }}$ for both of them is $\sim 5.7 \mathrm{mG}$. In the right middle panel we plot the $V$ spectrum for April and the predicted spectrum using a similar $B_{\mathrm{los}} \sim 5.5 \mathrm{mG}$. Even taking 
Table 7. Timetable of our MERLIN observations.

\begin{tabular}{cccc}
\hline \hline \multicolumn{2}{c}{ Starting time } & \multicolumn{2}{c}{ Ending time } \\
\hline 13-03-2008 & $03: 16$ & $13-03-2008$ & $10: 27$ \\
$07-04-2008$ & $01: 49$ & $07-04-2008$ & $09: 07$ \\
$08-04-2008$ & $01: 49$ & $08-04-2008$ & $09: 07$ \\
$04-07-2008$ & $19: 32$ & $05-07-2008$ & $02: 29$ \\
\hline
\end{tabular}

Table 8. Conversion table of the maser features, between our MERLIN observations, Walsh et al. (1998), and Goedhart et al. (2009).

\begin{tabular}{ccc}
\hline \hline This paper & Walsh et al. (1998) & Goedhart et al. (2009) \\
\hline F.01 39.2 & A 39.2 & 39.2 \\
F.02 38.8 & B 38.7 & 38.7 \\
F.03 37.7 & C 37.6 & 37.7 \\
F.04 36.4 & - & \\
- & D 36.6 & 36.6 \\
F.05 34.7 & E 34.6 & 34.6 \\
F.06 33.7 & F 33.6 & 33.6 \\
- & G 32.7 & 32.7 \\
- & H 31.6 & 31.6 \\
F.07 33.6 & - & - \\
F.08 32.7 & - & - \\
F.09 32.7 & - & - \\
F.10 38.2 & - & - \\
F.11 30.0 & J 30.0 & 30.0 \\
\hline
\end{tabular}

Notes. Velocities in $\mathrm{km} \mathrm{s}^{-1}$ are also reported for comparison.

into account an increased noise in the April observations, the circular polarisation spectrum is not consistent with the expected spectrum.

Another possible explanation could be to consider a change in the magnetic field direction between March and April. We show this case in Fig. 7, where the two hyperfine components present the inverse behaviour with respect to the previous case. The magnetic field is still $\left|B_{\text {los }}\right| \sim 5.5 \mathrm{mG}$ for both components. Similar variability of the magnetic field has previously been observed and the origin of this change can be intrinsic to the source as discussed by Vlemmings et al. (2009).

Finally, another possibility is to consider the two components as two different and blended masers, originating in two places lying along the same line of sight (e.g. Momjian \& Sarma 2017), and with varying intensity. In this case we can only measure the average magnetic field along the line of sight $B_{\text {los }} \sim 5.5 \mathrm{mG}$ in March, and $B_{\text {los }} \sim-4.9 \mathrm{mG}$ in April. We report $B_{\text {los }}$ for this last case in Tables 3 and 4.

However, it is impossible at this stage to discern which of these phenomena is occurring. Higher resolution observations covering more epochs could help to increase the signal-to-noise ratio and better distinguish the two components' contributions.

In all cases, the error on $B_{\text {los }}$ is approximately $30 \%$ and was estimated on the basis of the rms noise in the line-free channels of the $V$ spectrum. All the above values are comparable with $B_{\text {pos }} \sim 11 \mathrm{mG}$ already obtained by Beuther et al. (2010), from dust continuum polarisation observations. Considering $B_{\text {los }} \sim$ $5.5 \mathrm{mG}$, we can obtain the total magnetic field strength $B_{\mathrm{tot}} \sim$ $\sqrt{B_{\text {pos }}^{2}+B_{\text {los }}^{2}} \sim 12 \mathrm{mG}$ and since $B_{\text {tot }}=B_{\text {los }} / \cos \theta$ we can estimate the angle between the magnetic field and the line of sight $\theta=63_{-6}^{\circ+8}$. This angle is in agreement with our assumption that $\theta>\theta_{\text {crit }} \sim 55^{\circ}$ (see Sect. 4) and it is consistent with the strength of our measured polarisation fraction (e.g. Surcis et al. 2015).

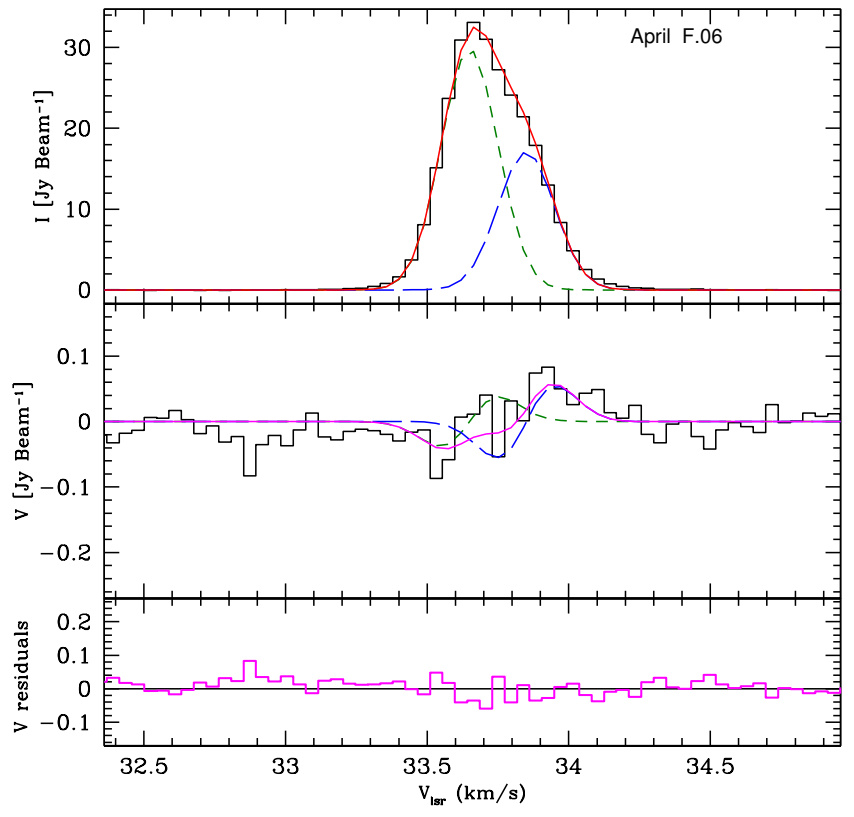

Fig. 7. Fit to maser feature F.06 assuming the presence of two hyperfine components, but with a magnetic field that changes its sign between March and April. Since the March panel is identical to that in Fig. 6, we only show the April panel. Panels and lines are as in Fig. 6.

\section{Conclusions}

In this paper, we present our investigation of the magnetic field morphology of the well-known high-mass-star forming region IRAS 18089-1732. We analysed a three-epoch MERLIN observation of the $6.7 \mathrm{GHz} \mathrm{CH}_{3} \mathrm{OH}$ maser generated in a region of a few au, close to the disc.

We identified nine masers in March and April and seven masers in July, confirming almost all the maser features already seen, as well as some new detections. The July observations were in dual circular polarisation only, so we performed the linear polarisation analysis only on the masers observed in the first two epochs, for which we measured the median linear polarisation fraction $\left(P_{1}\right)$ and the median linear polarisation angle $(\chi)$ across the spectrum. We identified two groups of masers on the basis of two different velocities and $\chi$ values: a blue group spanning a velocity range from 30.0 to $36.4 \mathrm{~km} \mathrm{~s}^{-1}$, and a red group from 37.7 to $39.2 \mathrm{~km} \mathrm{~s}^{-1}$.

The two groups of masers showed ordered linear polarisation vectors, and the orientation was preserved in both epochs. The blue group had a weighted average angle of $\chi_{\mathrm{B}, \mathrm{M}}=-24^{\circ} \pm 8^{\circ}$ in March and $\chi_{\mathrm{B}, \mathrm{A}}=-31^{\circ} \pm 12^{\circ}$ in April. The red group had only one linear polarised emission in March with an angle $\chi_{\mathrm{R}, \mathrm{M}}=$ $-78^{\circ} \pm 5^{\circ}$, while in April the weighted polarisation angle was $\chi_{\mathrm{R}, \mathrm{A}}=-70^{\circ} \pm 2^{\circ}$.

All three epochs were close to maxima if extrapolated from Goedhart et al. (2009) but for two of the epochs the brightest feature had a flux density much lower than predicted, suggesting irregular periodicity or a change in magnitude or both. Our positions, more accurate than those in Goedhart et al. (2009), confirmed the separations of features, and implied lower limits to light travel time that are in some cases incompatible with the simplest interpretations of time delays.

From the monitoring of Goedhart et al. (2009), we noticed that all the masers in the red group have variability with peaks occurring ahead of that of the reference feature, while those in the blue group lag behind. Since the two groups are separated in 
Table 9. Best-fit parameters for the two-component model for feature F.06.

\begin{tabular}{ccccccc}
\hline \hline Component & \multicolumn{2}{c}{ Intensity } & \multicolumn{2}{c}{ Centre velocity } & \multicolumn{2}{c}{$\begin{array}{c}\text { Velocity linewidth } \\
\left(\mathrm{km} \mathrm{s}^{-1}\right)\end{array}$} \\
& \multicolumn{2}{c}{$\left(\mathrm{Jy} \mathrm{beam}^{-1}\right)$} & \multicolumn{2}{c}{$\left(\mathrm{km} \mathrm{s}^{-1}\right)$} & \multicolumn{2}{c}{} \\
& March & April & March & April & March & April \\
\hline Component 1 & 35.50 & 17.00 & 33.87 & 33.85 & 0.11 & 0.10 \\
Component 2 & 16.50 & 29.50 & 33.67 & 33.65 & 0.09 & 0.10 \\
\hline
\end{tabular}

polarisation angles and velocities, we deduce that the two groups of masers are emitted by two different regions, one lying on the disc of the protostar and another closer to the base of the molecular outflow. Therefore we suggest they are probing two different magnetic field directions, with an orientation on the plane of the sky of $\Phi^{\text {disc }}=+62^{\circ} \pm 3^{\circ}$ and $\Phi^{\text {outflow }}=+14^{\circ} \pm 4^{\circ}$.

We showed that the small-scale magnetic field probed by the masers is consistent with the large-scale magnetic field traced by the dust (Beuther et al. 2010). Therefore we conclude that the large-scale field component, even at the au scale of the masers, dominates over any small-scale field fluctuations.

We proposed a tentative detection of circular polarisation for one of the brightest features, F.06. The shape of the total power and of the circular polarisation spectra appear to reverse between the March and the April observations. This could be due to the splitting of two hyperfine components, each one emitting preferentially in a different epoch. Another possibility could be that the magnetic field reverses its sign, as already suggested for another variable $6.7 \mathrm{GHz}$ maser. Yet another option could be that we observed two different masers, originating in two places lying along the same line of sight. In all the three cases we obtained a $\left|B_{\text {los }}\right| \sim 5 \mathrm{mG}$, comparable to $B_{\text {pos }} \sim 11 \mathrm{mG}$ already obtained by Beuther et al. (2010) for dust.

Acknowledgements. We thank the anonymous referee whose comments have contributed to improve the presentation of this paper. The research leading to these results has received funding from the European Research Council under the European Union's Seventh Framework Programme (FP/2007-2013)/ERC Grant Agreement No. 614264

\section{References}

Allen, A., Li, Z.-Y., \& Shu, F. H. 2003, ApJ, 599, 363

Banerjee, R., \& Pudritz, R. E. 2007, ApJ, 660, 479

Beltrán, M. T., \& de Wit, W. J. 2016, A\&ARv, 24, 6

Beuther, H., \& Walsh, A. J. 2008, ApJ, 673, L55

Beuther, H., Schilke, P., Menten, K. M., et al. 2002, ApJ, 566, 945

Beuther, H., Hunter, T. R., Zhang, Q., et al. 2004a, ApJ, 616, L23

Beuther, H., Zhang, Q., Hunter, T. R., et al. 2004b, ApJ, 616, L19

Beuther, H., Zhang, Q., Sridharan, T. K., \& Chen, Y. 2005, ApJ, 628, 800

Beuther, H., Vlemmings, W. H. T., Rao, R., \& van der Tak, F. F. S. 2010, ApJ, 724, L113

Bonnell, I. A., \& Bate, M. R. 2006, MNRAS, 370, 488

Cesaroni, R., Galli, D., Lodato, G., Walmsley, M., \& Zhang, Q. 2006, Nature, 444, 703

Cesaroni, R., Galli, D., Lodato, G., Walmsley, C. M., \& Zhang, Q. 2007, Protostars and Planets V, 197

Dodson, R., \& Moriarty, C. D. 2012, MNRAS, 421, 2395

Ellingsen, S. P. 2002, in Cosmic Masers: From Proto-Stars to Black Holes, eds. V. Migenes, \& M. J. Reid, IAU Symp., 206, 151

Girart, J. M., Beltrán, M. T., Zhang, Q., Rao, R., \& Estalella, R. 2009, Science, 324,1408

Goedhart, S., Minier, V., Gaylard, M. J., \& van der Walt, D. J. 2005, MNRAS, 356,839

Goedhart, S., Langa, M. C., Gaylard, M. J., \& van der Walt, D. J. 2009, MNRAS, 398,995
Goldreich, P., \& Kylafis, N. D. 1981, ApJ, 243, L75

Goldreich, P., \& Kylafis, N. D. 1982, ApJ, 253, 606

Green, J. A., Richards, A. M. S., Vlemmings, W. H. T., Diamond, P., \& Cohen, R. J. 2007, MNRAS, 382, 770

Isokoski, K., Bottinelli, S., \& van Dishoeck, E. F. 2013, A\&A, 554, A100

Krumholz, M. R., Crutcher, R. M., \& Hull, C. L. H. 2013, ApJ, 767, L11

Lankhaar, B., Groenenboom, G. C., \& van der Avoird, A. 2016, J. Chem. Phys., 145,244301

Lankhaar, B., Vlemmings, W. H. T., \& Surcis, G. 2017, Nature Astron., submitted

Leurini, S., Schilke, P., Wyrowski, F., \& Menten, K. M. 2007, A\&A, 466, 215

Li, Z.-Y., Krasnopolsky, R., \& Shang, H. 2011, ApJ, 738, 180

Li, Z.-Y., Krasnopolsky, R., \& Shang, H. 2013, ApJ, 774, 82

Li, H.-B., Goodman, A., Sridharan, T. K., et al. 2014, Protostars and Planets VI, 101

Machida, M. N., Inutsuka, S.-I., \& Matsumoto, T. 2014, MNRAS, 438, 2278

McKee, C. F., \& Tan, J. C. 2003, ApJ, 585, 850

Mellon, R. R., \& Li, Z.-Y. 2008, ApJ, 681, 1356

Mestel, L., \& Spitzer, Jr., L. 1956, MNRAS, 116, 503

Momjian, E., \& Sarma, A. P. 2017, ApJ, 834, 168

Myers, A. T., McKee, C. F., Cunningham, A. J., Klein, R. I., \& Krumholz, M. R. 2013, ApJ, 766, 97

Peters, T., Banerjee, R., Klessen, R. S., \& Mac Low, M.-M. 2011, ApJ, 729, 72

Reid, M. J., Schneps, M. H., Moran, J. M., et al. 1988, ApJ, 330, 809

Seifried, D., Banerjee, R., Klessen, R. S., Duffin, D., \& Pudritz, R. E. 2011, MNRAS, 417, 1054

Seifried, D., Banerjee, R., Pudritz, R. E., \& Klessen, R. S. 2012, MNRAS, 423, L40

Seifried, D., Banerjee, R., Pudritz, R. E., \& Klessen, R. S. 2015, MNRAS, 446 2776

Sridharan, T. K., Beuther, H., Schilke, P., Menten, K. M., \& Wyrowski, F. 2002, ApJ, 566, 931

Surcis, G., Vlemmings, W. H. T., Dodson, R., \& van Langevelde, H. J. 2009, A\&A, 506, 757

Surcis, G., Vlemmings, W. H. T., Curiel, S., et al. 2011, A\&A, 527, A48

Surcis, G., Vlemmings, W. H. T., van Langevelde, H. J., \& Hutawarakorn Kramer, B. 2012, A\&A, 541, A47

Surcis, G., Vlemmings, W. H. T., van Langevelde, H. J., et al. 2014a, A\&A, 565 , L8

Surcis, G., Vlemmings, W. H. T., van Langevelde, H. J., Moscadelli, L., \& Hutawarakorn Kramer, B. 2014b, A\&A, 563, A30

Surcis, G., Vlemmings, W. H. T., van Langevelde, H. J., et al. 2015, A\&A, 578, A102

Tan, J. C., Beltrán, M. T., Caselli, P., et al. 2014, Protostars and Planets VI, 149

Troland, T. H., \& Heiles, C. 1982, ApJ, 252, 179

Vlemmings, W. H. T. 2008, A\&A, 484, 773

Vlemmings, W., Diamond, P. J., \& van Langevelde, H. J. 2001, A\&A, 375, L1

Vlemmings, W. H. T., Diamond, P. J., \& van Langevelde, H. J. 2002, A\&A, 394, 589

Vlemmings, W. H. T., Diamond, P. J., van Langevelde, H. J., \& Torrelles, J. M. 2006, A\&A, 448, 597

Vlemmings, W. H. T., Goedhart, S., \& Gaylard, M. J. 2009, A\&A, 500, L9

Vlemmings, W. H. T., Surcis, G., Torstensson, K. J. E., \& van Langevelde, H. J. 2010, MNRAS, 404, 134

Vlemmings, W. H. T., Torres, R. M., \& Dodson, R. 2011, A\&A, 529, A95

Walsh, A. J., Burton, M. G., Hyland, A. R., \& Robinson, G. 1998, MNRAS, 301, 640

Wardle, J. F. C., \& Kronberg, P. P. 1974, ApJ, 194, 249

Xu, Y., Moscadelli, L., Reid, M. J., et al. 2011, ApJ, 733, 25

Zapata, L. A., Rodríguez, L. F., Ho, P. T. P., Beuther, H., \& Zhang, Q. 2006, AJ, 131,939

Zhao, B., Caselli, P., Li, Z.-Y., et al. 2016, MNRAS, 460, 2050 